\title{
Practical Aspects and Usage Tips for the Volta Phase Plate
}

\author{
Radostin Danev $^{1}$, Bart Buijsse ${ }^{2}$, Maryam Khoshouei ${ }^{1}$, Yoshiyuki Fukuda ${ }^{1}$, Wolfgang Baumeister ${ }^{1}$ \\ 1. Max Planck Institute of Biochemistry, Martinsried, Germany. \\ 2. FEI, Eindhoven, The Netherlands.
}

Compared to other technical advances in transmission electron microscopy (TEM), such as brighter electron sources, energy filters, better optics and direct detection cameras, phase plates have lagged behind in both development and applications. The main reason for that is the difficulty in solving practical issues, such as beam-induced electrostatic charging, which cannot be reliably predicted or avoided based on theoretical research. Another reason is that phase plates add more complexity to the operation of the microscope and until not so long ago were not usable for automated data acquisition.

The recently developed Volta phase plate (VPP) [1] solves most of the practical issues and is relatively easy to use and automate. It is already being routinely used in cryo-tomography applications where it has demonstrated remarkable improvements in contrast and visibility of features [2,3]. In single particle applications the VPP has shown some promise but the resolutions achieved thus have been limited to about $8 \AA$.

In this paper we present a few examples of the use of VPP in both cryo-tomography and single particle applications. In addition to the application results we will discuss the practical aspects of using a VPP. The most important microscope alignments for proper VPP operation are the on-plane condition and the beam shift pivots (Fig. 1).

The on-plane condition (Figs. 1a, b) concerns the position of the central beam crossover at the back focal plane relative to the phase plate. The VPP operates best on-plane, which provides a proper phase shift of $\sim \pi / 2$ (Fig. 2) and the lowest possible cut-on frequency. In suitably designed microscopes the onplane condition for the phase plate corresponds to parallel illumination on the specimen.

The beam shift pivot point setting (Figs. 1c, d) is responsible for the lateral stability of the beam crossover on the phase plate. When the pivot point is properly set the beam crossover remains stationary at a fixed point on the phase plate irrespective of the beam movements at the specimen plane. This setting is especially important for low-dose operation where beam shifts combined with image shifts are used to offset the observation area on the specimen for e.g. focusing.

In addition to the on-plane and beam shift pivot point alignments the third important factor for a successful VPP observation is proper conditioning of the phase plate. The phase shift of the VPP is created by the central beam and builds up gradually with the total dose on the phase plate. The blue curve in Fig. 2 can be used to calculate the pre-irradiation time required for creating a desired phase shift. The time in seconds is equal to the dose in $\mathrm{nC}$ (from the horizontal axis in Fig. 2) divided by the total beam current in $\mathrm{nA}$.

[1] R Danev et al, PNAS 111 (2014) p. 15635.

[2] S Asano et al, Science 23 (2015) p. 439.

[3] Y Fukuda et al, Journal of Structural Biology (2015) in press. 
(a)

$$
\text { On-plane }
$$

(parallel illumination) (b)

Off-plane

(non-parallel illumination)

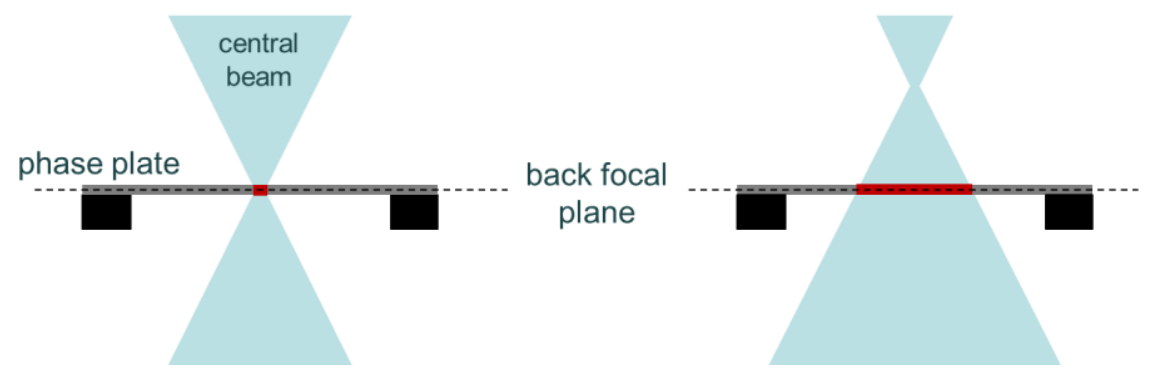

(c) Correct pivot point setting

(d) Incorrect pivot point setting

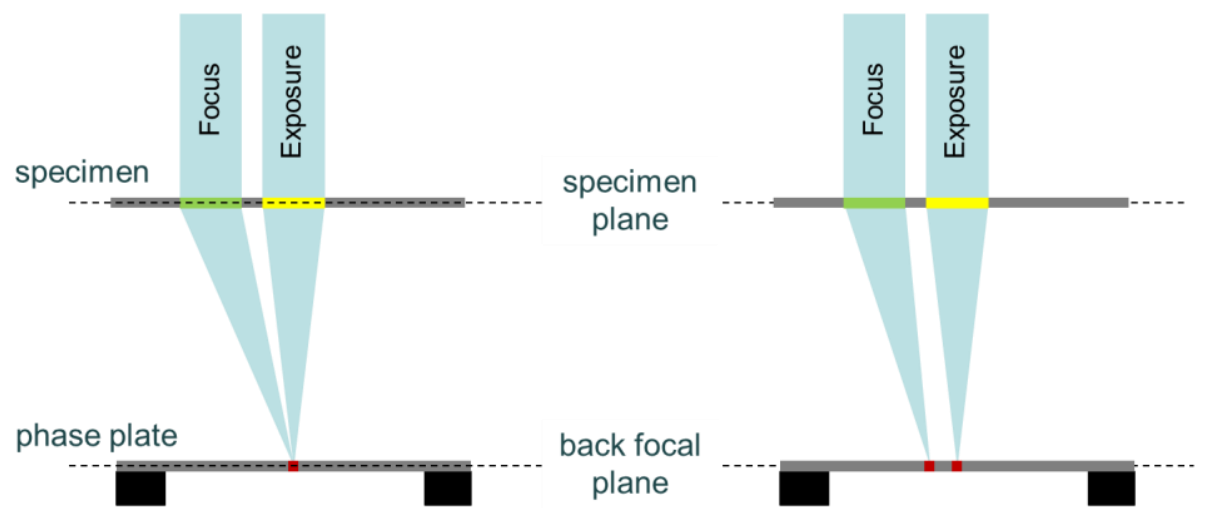

Figure 1. (a, b) On-plane and off-plane conditions. (c, d) Correct and incorrect setting of the beam shift pivot points.

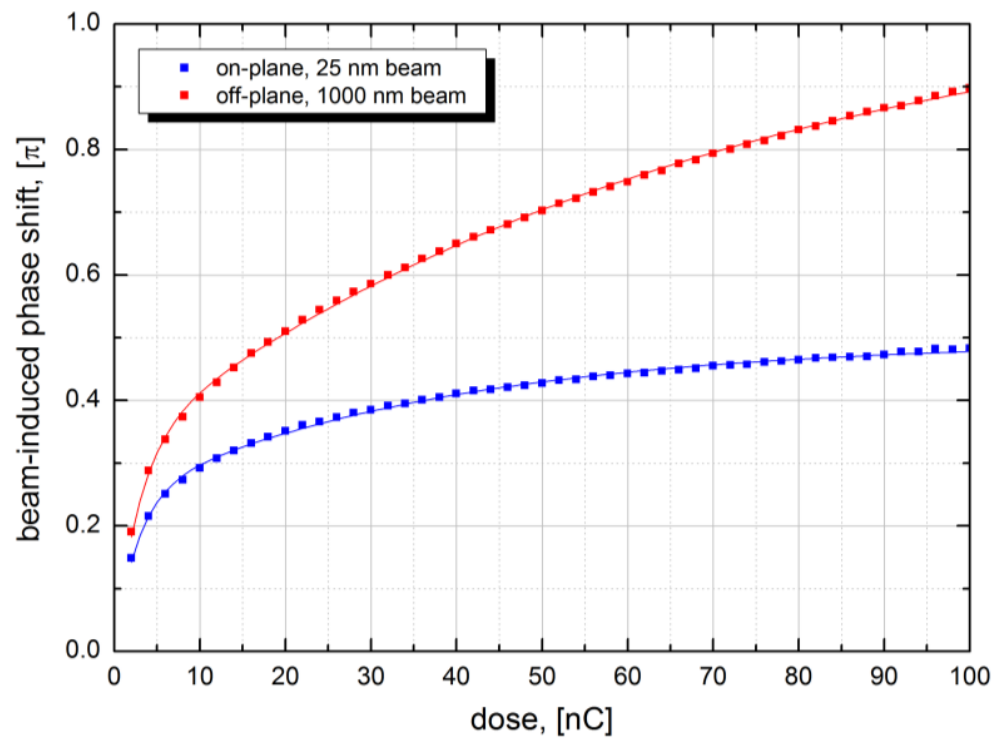

Figure 2. Phase shift vs. dose for the VPP on-plane (blue curve) and off-plane (red curve). 